\title{
Anomalous left coronary artery Report of an unusual case with spontaneous remission of symptoms
}

\author{
H. N. C. Ihenacho, S. P. Singh, R. Astley, and C. G. Parsons \\ From the Heart Unit and Radiology Department, The Children's Hospital, Birmingham
}

A case is reported of a I4-year-old girl with anomalous origin of the left coronary artery from the pulmonary artery. Symptoms were severe in childhood but there has been spontaneous remission since the age of Io.

Anomalous origin of the left coronary artery is a rare lesion in which the left coronary artery arises from the pulmonary artery and follows the peripheral course of the normal coronary artery. Most patients with this lesion begin showing symptoms during the neonatal period and are dead by the first year (Keith, Rowe, and Vlad, 1958). Only a few pass undiscovered during childhood until adult life when sudden death may occur (George and Knowlan, 1959). To our knowledge there are no reports where symptoms of angina and heart failure improved spontaneously. We wish to present the case report of a girl who had been severely handicapped during childhood but by the age of $\mathrm{I}_{4}$ had become asymptomatic.

\section{Case report}

This is a 14-year-old girl, born at full term by lower segment caesarean section because of prolonged labour. Pregnancy was normal. No abnormalities were detected at birth and she had a normal development during infancy. A cardiac murmur was an incidental finding at the age of 18 months. She remained asymptomatic and was referred to the Birmingham Children's Hospital at the age of 2 years for evaluation of the cardiac murmur. The heart was moderately enlarged. There was a pansystolic murmur at the apex but no accompanying thrill. Physical examination was otherwise normal. Radiography confirmed the presence of an enlarged heart due to a large left ventricle. The electrocardiogram showed obvious left ventricular hypertrophy. A right heart catheterization was performed at the age of 3 years: the pulmonary artery pressure was $20 / 8$ and wedge pressure was I0 $\mathrm{mmHg}$. Angiocardiography with contrast injection into the right ventricle showed a normal circulatory route. No conclusive diagnosis could be made.

A year later she developed exertional central chest pain which was relieved by rest. By the age of 6 years this symptom became more severe and was related to mild exertion such as walking up to 200 to 300 yards on the flat. She also became extremely breathless on moderate activity and had to be admitted for further investigation. Clinical examination then showed that she was orthopnoeic. The pulse was 90 a minute and rather large in volume. The blood pressure was $130 / 75 \mathrm{mmHg}$. The apex beat was in the 5th interspace and shifted to the left anterior axillary line. There was no evidence of cardiac failure. A loud pansystolic murmur was maximal at the apex and conducted to the axilla and there was a systolic thrill also best felt in the same area. There was a loud apical 3 rd sound and after this a mid diastolic murmur. The electrocardiogram showed increased left ventricular hypertrophy with tall $R$ waves in leads $V_{5}$ measuring $66 \mathrm{~mm}$. Deep $Q$ waves in aVL and left praecordial chest leads with ST depression led to the suspicion of anomalous origin of the left coronary artery or a primary cardiomyopathy (Fig. I and 2). Chest $x$-ray showed a much enlarged heart and pulmonary vascular congestion. Left and right heart catheterization was performed. This showed a pulmonary artery pressure of $40 / 15 \mathrm{mmHg}$. The left ventricular and diastolic pressure was $20-25 \mathrm{mmHg}$. Injection of contrast media into the left ventricle showed gross mitral incompetence. The right coronary artery was visualized and was found to be unusually large for a child: the origin of the left coronary artery from the aorta was not seen. The large right coronary artery and its branches in later stages of circulation filled the smaller left coronary artery from which the contrast medium passed into the main pulmonary trunk.

Because of her severe symptoms surgical treatment 


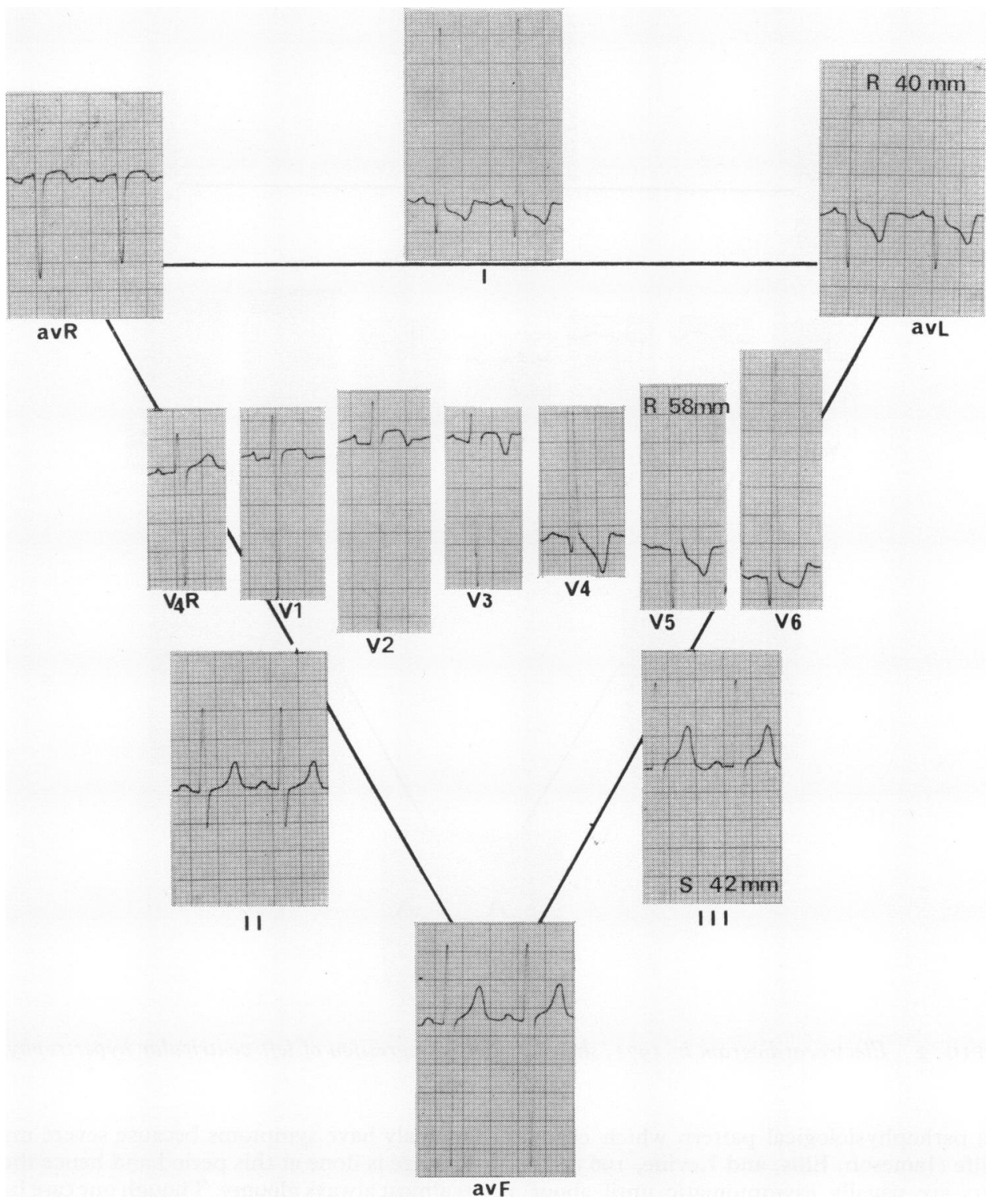

FIG. I Electrocardiogram in 1965, showing conspicuous left ventricular hypertrophy.

was discussed with the parents but they refused it. She remained severely disabled with angina and breathlessness until the age of ro years. Her symptoms then gradually improved and over the past two years she has become asymptomatic without any breathlessness or chest pain. She is now an active young girl who can take part in most of the school activities, including exercise such as swimming. The heart size is normal and this is confirmed by a normal chest $x$-ray. The systolic murmur has become very faint, is localized to the apex, and there is no accompanying thrill.

\section{Discussion}

Since Bland, White, and Garland described the clinical picture, including the electrocardiographic changes, of anomalous origin of the left coronary artery (Bland, White, and Garland, 1933), several cases have been reported. Attempts have been made to classify the clinical pictures into two groups: the more severe and commoner infantile group and the rarer 'adult' group (Gouley, 1950). However, it has also been suggested that this malformation may give 


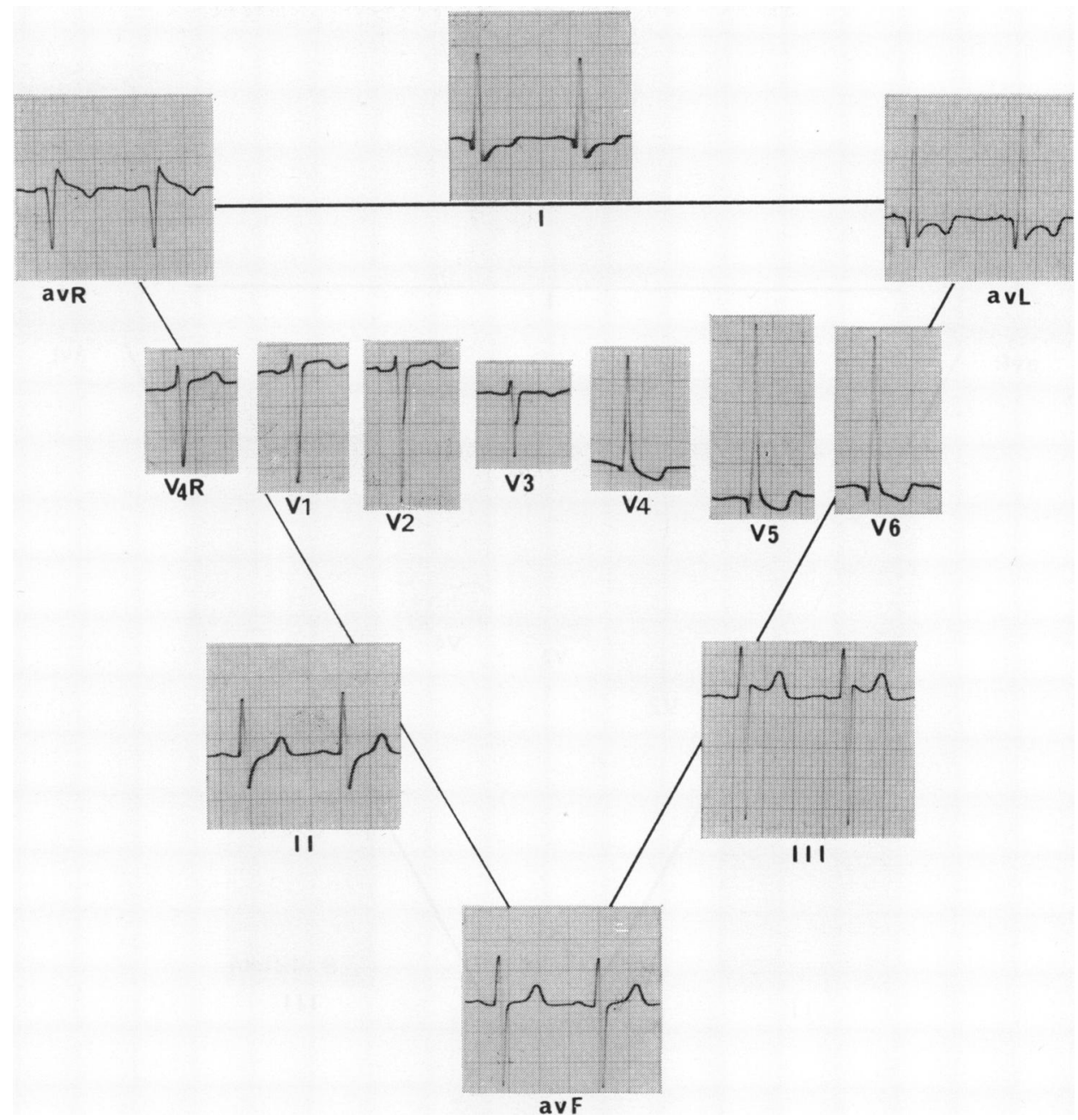

FIG. 2 Electrocardiogram in I97I, showing obvious regression of left ventricular hypertrophy.

rise to a pathophysiological pattern which changes during life (Jameson, Ellis, and Levine, 1963).

Infants are usually asymptomatic until about $\mathbf{I}$ month when feeding difficulties occur, with attacks of sweating, dyspnoea, cyanosis, and pallor associated with crying: all these appear to be precipitated by angina. Most of these babies die in congestive failure or suddenly from syncope. Mitral insufficiency is common and may be the main presentation, especially in adolescent and adult patients who were asymptomatic during childhood (Burchell and Brown, 1962). Edwards (1964) postulated that ischaemic change probably occurs in the transitional phase when pulmonary artery pressure drops below the foetal level and before intercoronary collaterals develop. Most of the infants with this anomaly have symptoms because severe myocardial damage is done at this period and hence the outlook is almost always gloomy. Though our case had severe symptoms of angina and mitral incompetence, she has spontaneously become asymptomatic, the signs of mitral incompetence are less, and the heart size normal. It is possible that our patient established sufficient collateral circulation to avert permanent damage.

It has been suggested that mitral insufficiency may lead to some beneficial adjustment by causing pulmonary hypertension which may lead to reversal of flow, thus allowing better perfusion of the damaged myocardium from the pulmonary artery, though with blood of low oxygen saturation (Foster et al., 1964). The usual direction of flow 
would allow run-off of blood into the pulmonary artery, giving an arteriovenous fistulous effect, with blood bypassing a great portion of the cardiac muscle. There is no clinical evidence of pulmonary hypertension in our patient. Thrombosis of the origin of the left coronary artery from the pulmonary artery would have the same effect as operative ligation of the origin of the anomalous left coronary artery, an operation that has been recommended when the direction of the flow has been shown to be from the coronary artery into the pulmonary artery (Nadas, Gamboa, and Hugenholtz, 1964). Here again we have no evidence of this complication in our patient.

As operation is not being considered in our patient, further catheterization is not thought justifiable and we may not know the exact cause of her spontaneous improvement.

\section{References}

Bland, E. F., White, P. D., and Garland, J. (1933). Congenital anomalies of the coronary arteries. Report of an unusual case associated with cardiac hypertrophy. American Heart fournal, 8, 787.
Burchell, H. B., and Brown, A. L. (1962). Anomalous origin of coronary artery from pulmonary artery masquerading as mitral insufficiency. American Heart fournal, 63, 388.

Edwards, J. E. (1964). Editorial: The direction of blood flow in coronary arteries arising from the pulmonary trunk. Circulation, 29, 163.

Foster, H. R., Hagstrom, J. W. C., Ehlers, K. H., and Engle, M. A. (1964). Mitral insufficiency due to the anomalous origin of the left coronary artery from the pulmonary artery. Pediatrics, 34, 649.

George, J. M., and Knowlan, D. M. (1959). Anomalous origin of the left coronary artery from the pulmonary artery in an adult. New England fournal of Medicine, 261, 993.

Gouley, B. A. (1950). Anomalous origin of left coronary artery from the pulmonary artery (adult type). American Heart Fournal, 40, 630 .

Jameson, A. G., Ellis, K., and Levine, O. R. (1963). Anomalous left coronary artery arising from pulmonary artery. British Heart fournal, 25, 251.

Keith, J. D., Rowe, R. D., and Vlad, P. (1958). Heart Disease in Infancy and Childhood. Macmillan, New York.

Nadas, A. S., Gamboa, R., and Hugenholtz, P. G. (1964). Anomalous left coronary artery originating from the pulmonary artery. Report of two surgically treated cases with a proposal of hemodynamic and therapeutic classification. Circulation, 29, 167.

Requests for reprints to Dr. C. G. Parsons, Heart Unit, The Children's Hospital, Ladywood Middleway, Ladywood, Birmingham, BI6 8ET. 